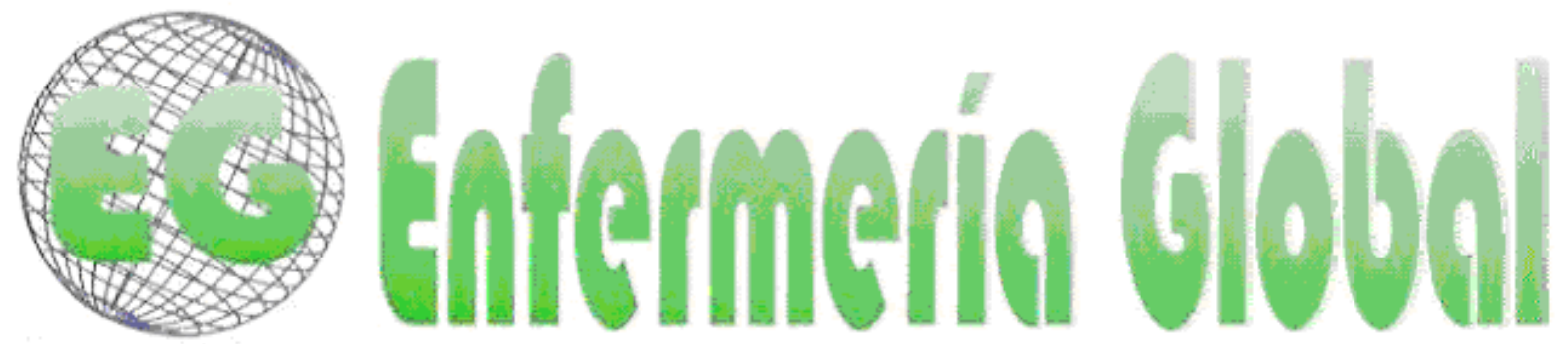

\title{
Factores intrínsecos predisponentes a infección hospitalaria y a óbito neonatal
}

Fatores intrínsecos predisponentes a infecção hospitalar e ao óbito neonatal The underlying intrinsic factors of infections at hospital and perinatal death

\section{${ }^{*}$ Chaves Ribeiro, lara ${ }^{* *}$ Costa Aguiar, Beatriz Gerbassi}

\author{
*Máster en Enfermería. E-mail: iaracribeiro@ig.com.br **Doctora en Enfermería. Escuela de \\ Enfermería Alfredo Pinto. Universidad Federal del Estado de Rio de Janeiro - UNIRIO. Brasil.
}

\begin{abstract}
Palabras clave: Recién nacido; óbito neonatal; Unidad de terapia intensiva neonatal
Palavras-chave: Recém-nascido; Óbito neonatal; Unidade de terapia intensiva neonatal

Keywords: Newborn; perinatal deaths; neonatal intensive care unit.
\end{abstract}

\section{RESUMEN}

Estudiar en las historias clínicas de los recién nacidos que evolucionaron a muerte en la UCI, objetivando conocer los factores de riesgo intrínsecos que predisponen a la infección nosocomial en los casos de muerte neonatal. Los factores de riesgo estudiados fueron: sexo, peso al nacer, edad gestacional, test de Apgar, y la rotura de membranas. Estudio descriptivo, retrospectivo, con un enfoque cuantitativo. En este estudio se encontró $55,2 \%$ de las muertes en el recién nacido de sexo femenino; en lo referente al peso al nacer, entre los 29 registros de defunciones consultados, el 48,3\% de los recién nacidos pesaron menos de 750g; respecto a la puntuación de Apgar en el 5 minuto $37,9 \%$ de los recién nacidos no presentó asfixia. En la evaluación de la edad gestacional, se verificó que el 37,9\% de los óbitos se encontraba entre 25-27 semanas; en relación con la rotura de bolsa, $62 \%$ de los partos con bolsa rota en el acto, 20,7\%, con bolsa rota más de 24 horas. El estudio muestra la importancia en el cuidado de la mujer durante el embarazo hasta el parto. El bajo peso y baja edad gestacional son riesgos inherentes de extrema importancia en la predisposición de los recién nacidos a complicaciones que puede llevarlos a la muerte.

\section{RESUMO}

Estudo realizado em prontuários de recém-nascidos que evoluíram ao óbito na UTIN, objetivando conhecer os fatores de risco intrínsecos predisponentes a infecção hospitalar na ocorrência do óbito neonatal. Os fatores de risco estudados foram: sexo, peso de nascimento, idade gestacional, escore de apgar e tempo de bolsa rota. Pesquisa descritiva, retrospectiva, com abordagem quantitativa. Neste estudo foram encontrados $55,2 \%$ dos óbitos nos recém-nascidos do sexo feminino; no peso de nascimento, dentre os 29 prontuários de óbitos pesquisados, 48,3\% dos recém-nascidos tinham peso inferior a 750g; no que diz respeito ao índice de apgar no $5^{\circ}$ minuto 37,9\% dos recém-nascidos não apresentaram asfixia; na avaliação da idade gestacional, pode-se verificar que 37,9\% dos óbitos se encontravam entre $25-27$ semanas; com relação ao tempo de bolsa rota, foram encontrados $62 \%$ dos 
partos com bolsa rota no ato, $20,7 \%$ com bolsa rota superior às $24 \mathrm{~h}$. O estudo mostra a importância nos cuidados prestados a mulheres durante a gravidez até o parto. O baixo peso e a baixa idade gestacional são riscos intrínsecos de extrema importância na predisposição dos recém-nascidos a complicações podendo consequentemente levá-los ao óbito.

\section{ABSTRACT}

To study the medical record of the new born babies that were dead in the ICU in order to know the underlying risk factors that influence to the hospital infections in the neonatal death cases. The studied risk factors were the following: sex, weight, gestational age, Apgar test and the rupture of the membranes. This is a descriptive study, retrospective with a quantitative approach. In this study, it was found a $55,2 \%$ of deaths in the female sex new born. Referring to the weight, from the 29 registers of the consulted deaths, the $48,3 \%$ of the new born weighed less than $750 \mathrm{~g}$; regarding the marks at Apgar in the 5th minute $37,9 \%$ of the new born did not present any suffocation. In the evaluation of the gestational age, it was verified that the $37,9 \%$ of the deaths were between $25-27$ weeks; in relation to the breaking of the bag of water, the $62 \%$ of the labours with broken bag of water at that moment, the $20,7 \%$ with the bag broken more than 24 hours. The study shows the importance in the care of women during the pregnancy until the labour. The underweight and the low gestational age are inherent risks of extreme importance in the predisposition of new born to complications that can provoke them their death.

\section{INTRODUCCIÓN}

Este estudio es un extracto de la Disertación de Máster en Enfermería, Escuela de Enfermería Alfredo Pinto Universidad Federal del Estado de Rio de Janeiro- UNIRIO, defendida en marzo de 2010, que aborda el tema de las muertes de recién nacidos con asociaciones infecciosas- la enfermera investigando los riesgos inherentes.

Las complicaciones que surgen durante el embarazo pueden poner en riesgo el crecimiento fetal, lo cual contribuye al nacimiento antes de tiempo y por lo tanto a la hospitalización de los recién nacidos en la unidad de cuidados intensivos neonatales (UCIN).

Muchos son los factores de riesgo que predisponen a los recién nacidos cuando son ingresados en la UCIN, sobre todo los riesgos relativos a su maduración, lo que podría aumentar la posibilidad de complicaciones que a veces pueden ser fatales en este grupo de edad.

Por lo tanto, los factores de riesgo intrínsecos se refieren a la inmadurez en el desarrollo del sistema inmune, incluyendo características relacionadas específicamente con el paciente / huésped, es decir, tipo de gravedad de la enfermedad subyacente, el estado nutricional, la edad, el uso de fármacos inmunosupresores, entre otros ${ }^{(1)}$.

La asistencia ofrecida a los recién nacidos ingresados en la UCIN de riesgo se asocia con una alta morbilidad y mortalidad, lo que requiere una atención myor del equipo interdisciplinario y actualizar sus habilidades y conocimientos científicos.

Los bebés prematuros son los principales usuarios de la UCIN, reciben los procedimientos más invasivos, pasan un largo período en el hospital, por lo tanto, parecen predispuestos a complicaciones infecciosas graves que pueden conducir a la muerte neonatal, lo que refleja el aumento del número de muertes por causas prevenibles. 
Una parte de las muertes infantiles se pueden prevenir, por lo que conocer los factores de riesgo es importante para que podamos actuar con anticipación para evitar que el recién nacido muera.

Infecciones hospitalarias son consideradas evitables, y un desafío para los profesionales de la salud, especialmente los que trabajan en el cuidado de UCIN para recién nacidos extremadamente frágiles, donde se considera cualquier tipo de agresión un riesgo potencial para el sistema inmunológico.

La prematuridad y el bajo peso al nacer son factores intrínsecos que predisponen a la infección nosocomial. Cuanto mayor sea el peso, menor es el grado de prematuridad, más eficaz es la capacidad para proteger el cuerpo del recién nacido.

En un estudio ${ }^{(2)}$ identificar los factores de riesgo de mortalidad neonatal en la ciudad de Maringá-PR, en 2003 y 2004, mostró que los recién nacidos de bajo peso al nacer tenían un riesgo de muerte $44,10 \%$ mayor que los nacidos con peso normal.

Se observa en las UCIN's que los recién nacidos con mayor peso y edad gestacional tienen menos complicaciones durante la hospitalización, sin embargo, aquellos con menor peso tiene una mayor fragilidad y el riesgo consiguiente de aumento de la mortalidad.

En la medida en que aumenta la supervivencia de los niños prematuros de bajo peso al nacimiento y la edad gestacional, aumenta el riesgo de contraer enfermedades relacionadas con el tiempo de exposición en la unidad y las infecciones hospitalarias (3-4)

Un estudio ${ }^{(5)}$ en la unidad neonatal del Hospital Nossa Senhora da Conceição, en Santa Catarina, mostró que la tasa más alta (46\%) de sepsis neonatal (hasta el 7 ำ día de vida) fue en los recién nacidos con edad gestacional entre 29 y 34 semanas de gestación, evidenciando la prematuridad como un factor de riesgo importante de infección nosocomial.

Para los autores ${ }^{(6)}$, el grado de inmunodeficiencia está directamente asociado con la edad gestacional. Todo recién nacido es inmunológicamente deficiente debido a que su organismo presenta una debilidad en el sistema de defensa. Además, las complicaciones de la prematuridad aumentan el riesgo de infección nosocomial.

El tema de la infección hospitalaria neonatal se ha discutido no sólo por las autoridades, sino también entre los equipos que trabajan en la UCIN, convirtiéndose en el objeto de estudio y de investigación de los profesionales ${ }^{(7)}$.

Se observa que los profesionales de la salud tratan de mantener la mejor calidad de la atención en la unidad, una comprensión clara de su enfoque en el cliente a su cuidado, especialmente con respecto a las prácticas de prevención y control de la infección hospitalaria.

Este estudio pretende contribuir a la reflexión, señalando cuestiones importantes para la investigación de los factores de riesgo intrínsecos de los recién nacidos que predisponen a complicaciones infecciosas, con el fin de conocer los factores de riesgo intrínsecos que predisponen a la infección nosocomial en la ocurrencia del óbito neonatal. 


\section{MATERIAL Y MÉTODO}

Se trata de un estudio descriptivo, retrospectivo, de las muertes de recién nacidos asociadas con infección que ocurrieron entre junio de 2006 y junio de 2009 en la unidad de cuidados intensivos de un hospital universitario pertenenciente a la Secretaría de Salud del Estado de Río de Janeiro.

La muestra del estudio estuvo integrada por las muertes de recién nacidos, hasta los 28 días de vida, prematuros, con un máximo de 37 semanas de edad gestacional, que fueron ingresados en la UCIN de junio 2006 a junio de 2009.

El estudio excluyó las muertes de recién nacidos con una edad gestacional mayor de 37 semanas, y los que fueron ingresados en la UCIN durante más de 28 días.

La recolección de datos se realizó a través de lista de control donde los siguientes factores de riesgo fueron identificados en los registros: sexo, peso al nacer, la edad gestacional, Apgar y la puntuación de la rotura de membranas.

El proyecto fue revisado por el comité de ética del hospital y de investigación en el estudio y fue aprobado bajo el número de protocolo 2324 HUPE.

\section{RESULTADOS Y DISCUSIÓN}

Evaluación del recién nacido que falleció en los primeros 5 días de hospitalización, se observa que el $61,5 \%$ de los varones recién nacidos no resistió más de 5 días de vida, mientras que entre los recién nacidos del sexo femenino, el 37,5\% no sobreviven más de 5 días en la UCIN.

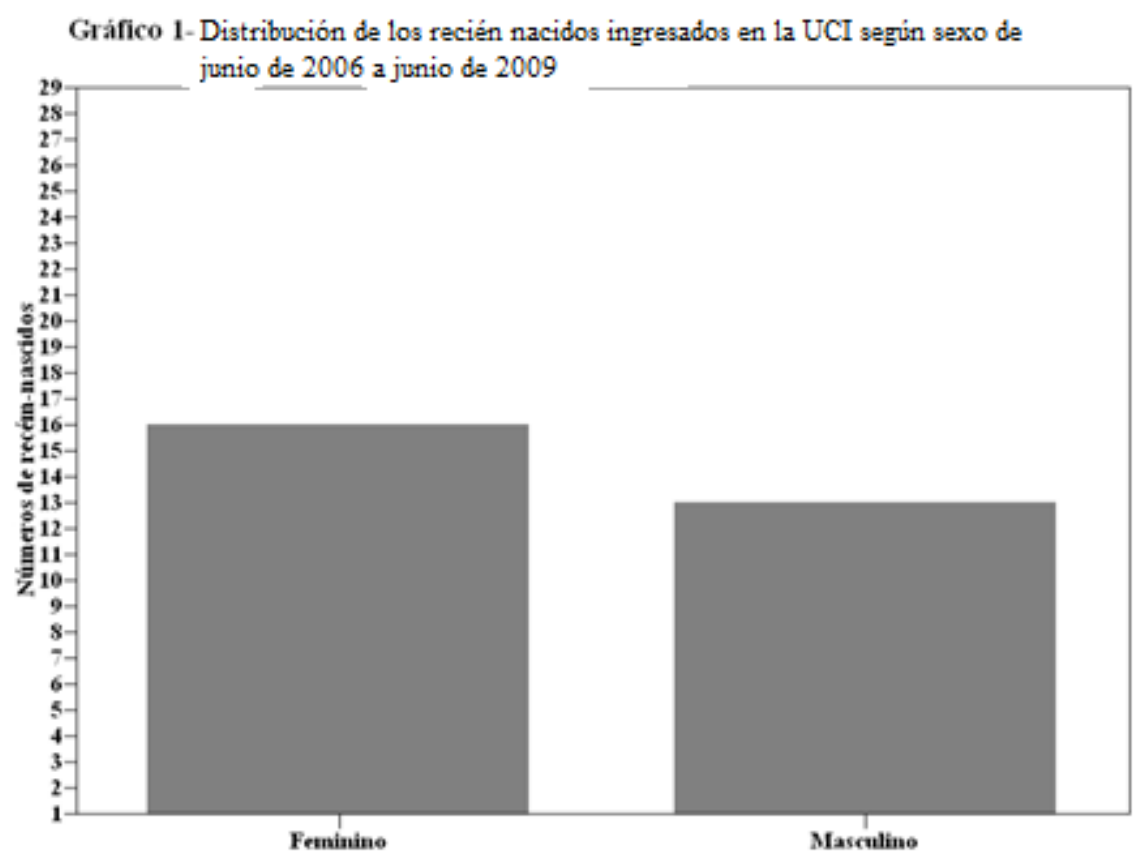

Los estudios han demostrado que los recién nacidos de sexo masculino tienen una mayor fragilidad y evolucionaron a la muerte en los primeros días de vida. Autores ${ }^{(8)}$, reafirman el predominio del sexo masculino en un estudio de evaluación de la atención de salud a través de la investigación de las muertes infantiles, informó que el 
$66 \%$ de los fallecidos eran de sexo masculino, mientras que los femeninos son alrededor de $31,9 \%$.

En este estudio, la duración media de ingreso de sexo mascuilno fue de 6 días, y de sexo femenino de 9 días. Este hecho confirma la explicación de los autores con respecto a la mayor resistencia del sexo femenino en comparación con el masculino.

En el análisis del peso al nacer, entre los 29 registros de muertes encuestados, el $48,3 \%$ de los recién nacidos tuvo peso inferior a $750 \mathrm{~g}$. Este resultado es similar al encontrado por los autores ${ }^{(9)}$, quienes en sus estudios mostraron un mayor número de muertes en recién nacidos que pesan menos de $749 \mathrm{~g}$. Estos datos refuerzan la idea de los riesgos existentes en este grupo de niños. Por otra parte, el $20,7 \%$ de los niños que progresaron a la muerte tuvieron peso al nacer entre 1501-2500g.

Tabla 1. Distribución de las defunciones de recién nacidos ingresados en la UCIN de junio 2006 a junio de 2009, de acuerdo con el peso al nacer.

\begin{tabular}{|l|l|l|l|}
\hline Peso al nacer & Frecuencia & Porcentaje & $\begin{array}{l}\text { Representación } \\
\text { gráfica }\end{array}$ \\
\hline$<\mathbf{7 5 0 g}$ & 14 & $48,3 \%$ & \\
\hline $\mathbf{7 5 1 - 1 0 0 0 g}$ & 5 & $17,3 \%$ & \\
\hline $\mathbf{1 0 0 1 - 1 5 0 0 g}$ & 3 & $10,3 \%$ & \\
\hline $\mathbf{1 5 0 1 - 2 5 0 0 g}$ & 6 & $20,7 \%$ & \\
\hline$>\mathbf{2 5 0 0 g}$ & 1 & $3,4 \%$ & \\
\hline Total & 29 & $100,0 \%$ & \\
\hline
\end{tabular}

Fuente: Datos de la encuesta (2009)

Este hecho nos lleva a una reflexión sobre la calidad de los procedimientos de atención y cuidado a los recién nacidos prematuros considerando las características específicas de su cuerpo.

Este autor ${ }^{(4)}$ dice que en los países desarrollados recién nacidos con peso entre 1000 y1500g tienen una tasa de supervivencia superior al $90 \%$, entre 751 y $1000 \mathrm{~g}, 86 \%$, y el $54-70 \%$ de los recién nacidos un peso inferior a $751 \mathrm{~g}$.

Las tasas de supervivencia de estos niños se diferencian entre las unidades, siendo evidente la importancia de la calidad de la atención a los recién nacidos en los primeros días de su vida. La evaluación de estas muertes es importante porque refleja la calidad de la atención prestada a estos niños durante la hospitalización, lo que sugiere cambios en los patrones establecidos.

Con respecto a la tasa de Apgar de los recién nacidos, el grado de asfixia evaluado a los 5 minutos se clasificó de la siguiente manera: el 37,9\% de los recién nacidos no mostró asfixia (8 a 10), el 41,4\% tenía grados de asfixia leve (5 a 7) y 20,7\% tenía leve asfixia (3 a 4). No había ningún bebé con grado severo de asfixia (0 a 2).

El índice de evaluación de la puntuación de Apgar es importante porque nos muestra si los recién nacidos precisaron intervenciones y procedimientos de un mayor nivel de complejidad, lo que puede significar mayores posibilidades de complicaciones y secuelas $^{(10)}$. 
Este estudio muestra que el 37,9\% de los recién nacidos no requería intervención y $41.4 \%$ tienen a asfixia leve, pudiendo haber sido necesario el uso de algún tipo de apoyo fundamental para su recuperación, lo que depende de la agilidad en la atención del parto y después del nacimiento.

Al analizar la variable de la edad gestacional, se encontró que el 37,9\% de las muertes se produjero en el rango de 25-27 semanas de edad gestacional, pero también fue la faja con mayor frecuencia, lo que se confirma por algunos autores ${ }^{(5)}$ cuando dicen que la edad gestacional es un factor importante tanto para la infección hospitalaria como la mortalidad neonatal.

Tabla 2-Distribución de las muertes de recién nacidos ingresados en la unidad de cuidados intensivos neonatales de junio 2006 a junio de 2009, de acuerdo con la edad gestacional.

\begin{tabular}{|l|l|l|l|}
\hline Edad Gestacional & Frecuencia & Porcentaje & Representación gráfica \\
\hline$<24$ & 3 & $10,3 \%$ & \\
\hline $\mathbf{2 5 - 2 7}$ & 11 & $37,9 \%$ & \\
\hline $\mathbf{2 8 - 3 0}$ & 6 & $20,7 \%$ & \\
\hline $\mathbf{3 1 - 3 3}$ & 4 & $13,8 \%$ & \\
\hline $\mathbf{3 4 - 3 7}$ & 4 & $13,8 \%$ & \\
\hline ignorado & 1 & $3,4 \%$ & \\
\hline Total & 29 & $100,0 \%$ & \\
\hline
\end{tabular}

Fuente: Datos de la encuesta (2009)

Con los avances tecnológicos, la supervivencia de los recién nacidos con una edad gestacional menor de 37 semanas es cada vez mayor, ya que tiempo atrás muchos de ellos no sobrevivirían.

Cuanto mayor es la edad gestacional, menor es el riesgo de muerte y también la duración de la estancia, que es un componente importante de la exposición de los recién nacidos a varias complicaciones, incluyendo infecciones nosocomiales.

Según este autor ${ }^{(11)}$, la mortalidad neonatal en niños con peso muy bajo al nacer sigue siendo elevada en nuestro país, especialmente en niños menores de $750 \mathrm{~g}$ y 29 Semanas de edad gestacional.

En este estudio, el $69 \%$ de las muertes fue en recién nacidos con una edad gestacional igual o inferior a 30 semanas, estos datos son significativos en comparación con los recién nacidos con una edad gestacional mayor de 30 semanas.

Este resultado se compara con muchos unidades neonatales de cuidados intensivos y con el estudio ${ }^{(11)}$, en el que los autores encontraron un porcentaje de $57,4 \%$ de las muertes de recién nacidos con edad gestacional menor de 29 semanas en un estudio para evaluar el riesgo de mortalidad neonatal.

Estos autores ${ }^{(12)}$ inciden en que la evaluación de la edad gestacional permite la observación de patrones anormales de crecimiento fetal. Y cuanto mayor sea la edad gestacional, mejor será la respuesta del cuerpo a cualquier tipo de terapia aplicada 
En relación a la ruptura de membranas, este estudio encontró un $62 \%$ de los nacimientos, con ruptura en el acto, del $20,7 \%$ con rotura superior a 24 horas, el $13,8 \%$, con rotura de menos de 24 horas y un 3,4\% no tenía identificación.

El estudio de la rotura de las membranas de los recién nacidos que progresaron a la muerte es importante porque podría indicar los factores relacionados con las causas de las muertes. Estas informaciones pueden ayudar a descartar y esclarecer factores que pueden estar directamente insertos en la asistencia.

\section{CONCLUSIÓN}

El conocimiento de los factores de riesgo intrínsecos de los recién nacidos prematuros que murieron con asociación con infección neonatal fue el foco principal de la investigación.

El estudio muestra la importancia de la atención a las mujeres durante el embarazo, a sabiendas de que éstas, si se desarrolla correctamente, predispone a un parto a término de recién nacidos capaces de mantener sus funciones vitales adecuadas.

Hacemos hincapié en la importancia de la salud pública para la provisión de una atención primaria de calidad desde la gestación hasta el parto, buscando una mayor adherencia de las mujeres a la atención prenatal, lo que refleja que un menor número de mujeres con complicaciones en el embarazo puede evitar poner en peligro la salud de las mujeres y los recién nacidos, debido principalmente a la disminución en el número de nacimientos de recién nacidos prematuros.

Con el creciente número de recién nacidos prematuros ingresados en la UCIN, mayores son las complicaciones adquiridas en la estancia en la unidad. El bajo peso y la baja edad gestacional son puntos muy importantes en la predisposición a estas complicaciones, que pueden llevarlos a la muerte.

El conocimiento de los factores de riesgo asociados con la prematuridad y la predisposición para el desarrollo de la infección nosocomial es importante para establecer una adecuada planificación de la atención, promoviendo así una mejor atención de calidad con el menor riesgo posible.

\section{REFERENCIAS}

1. Andrade, D E Angerami ELS. Reflexões acerca das infecções hospitalares às portas do terceiro milênio - Medicina, Ribeirão Preto, 32:492-497, Out/Dez. 1999.

2. Maran, E; Uchimura, T.T. Mortalidade Neonatal: fatores de risco em um município no sul do Brasil. Revista Eletrônica de Enfermagem [Internet] 2008;10(1):29-38. Available from: http://www.fen.ufg.br/revista/v10/n1/v10n1a03.htm.

3. Cucolo Danielle Fabiana, Faria Josimerci Ittavo Lamana, Cesarino Claudia Bernardi. Avaliação emancipatória de um Programa Educativo do Serviço de Controle de Infecção Hospitalar. Acta paul. enferm. [periódico na Internet]. 2007 Mar [citado 2010 Jun 26] ; 20(1): 49-54. Disponível em: http://www.scielo.br/scielo.php?script=sci_arttext\&pid=S0103$21002007000100009 \&$ Ing=pt. doi: $10.15 \overline{9} 0 /$ S0103-21002007000100009.

4. Prigenzi Maria Laura H., Trindade Cleide E.P., Rugolo Ligia M. S. S., Silveira Liciana V.A.. Fatores de risco associados à mortalidade de recém-nascidos de muito baixo peso na cidade de Botucatu, São Paulo, no período 1995-2000. Rev. Bras. 
Saude Mater. Infant. [periódico na Internet]. 2008 Mar [citado 2010 Jun 26] ; 8(1): 93-101. Disponível em: http://www.scielo.br/scielo.php?script=sci_arttext\&pid=S1519$38292008000100011 \&$ Ing=pt. doi: 10.1590/S1519-38292008000100011.

5. Goulart Ana Paula, Valle Caroline Fraga, Dal-Pizzol Felipe, Cancelier Ana Carolina Labor. Fatores de risco para o desenvolvimento de sepse neonatal precoce em hospital da rede pública do Brasil. Rev. bras. ter. intensiva [periódico na Internet]. 2006 Jun [citado 2010 Jun 26] ; 18(2): 148-153. Disponível em: http://www.scielo.br/scielo.php?script=sci_arttext\&pid=S0103-

507X2006000200008\&lng=pt. doi: $10.1590 /$ S0103-507X2006000200008.

6. Mussi-Pinhata Marisa M., Rego Maria A. C.. Particularidades imunológicas do prétermo extremo: um desafio para a prevenção da sepse hospitalar. J. Pediatr. (Rio J.) [periódico na Internet]. 2005 Mar [citado 2010 Jun 26] ; 81(1): . Disponível em: http://www.scielo.br/scielo.php?script=sci_arttext\&pid=S0021-

$75572005000200008 \&$ Ing=pt. doi: $10.1590 /$ S0021-75572005000200008.

7. Perez, C. Antimicrobianos em Unidades de Cuidados Intensivos: Uso empírico. Rev. Chil. Infect 2003; 20 (supl 1): 570-573.

8. Formigli Vera L. Almeida, Silva Lígia Maria Vieira da, Cerdeira Alfonso J. P., Pinto Cristiane M. F., Oliveira Renata S. A., Caldas Alessandra C. et al . Avaliação da atenção à saúde através da investigação de óbitos infantis. Cad. Saúde Pública [periódico na Internet]. [citado 2010 Jun 26]. Disponível em: http://www.scielo.br/scielo.php?script=sci_arttext\&pid=S0102-

311X1996000600005\&lng=pt. doi: 10.1590/S0102-311X1996000600005.

9. Duarte José Luiz Muniz Bandeira, Mendonça Gulnar Azevedo Silva. Avaliação dos óbitos neonatais em recém-nascidos de muito baixo peso em quatro maternidades no Município do Rio de Janeiro, Brasil. Cad. Saúde Pública [periódico na Internet]. 2005 Abr [citado 2010 Jun 26] ; 21(2): 387-395. Disponível em: http://www.scielo.br/scielo.php?script=sci_arttext\&pid=S0102-

311 X2005000200005\&lng=pt. doi: 10.1590/S0102-311X2005000200005.

10. Ramos, Helena Ângela de Camargo; Cuman Roberto Kenji Nakamura. Fatores de risco para prematuridade: Pesquisa documental. Esc Anna Nery Ver Enferm 2009 abrjun; 13 (2): 297-304.

11. Brito Angela Sara J de, Matsuo Tiemi, Gonzalez Maria Rafaela C, Carvalho Ana Berenice R de, Ferrari Lígia S L. Escore CRIB, peso ao nascer e idade gestacional na avaliação do risco de mortalidade neonatal. Rev. Saúde Pública [periódico na Internet]. 2003 Out [citado 2010 Jun 26] ; 37(5): 597-602. Disponível em: http://www.scielo.br/scielo.php?script=sci_arttext\&pid=S0034-

$89102003000500008 \&$ Ing=pt. doi: $10.1590 /$ S0034-89102003000500008.

12. Egewarth Cristiane, Pires Fernanda Dias Almeida, Guardiola Ana. Avaliação da idade gestacional de recém-nascidos pré-termo através do exame neurológico e das escalas neonatais e obstétrica. Arq. Neuro-Psiquiatr. [periódico na Internet]. 2002 Set [citado 2010 Jun 26] ; 60(3B): 755-759. Disponível em: http://www.scielo.br/scielo.php?script=sci_arttext\&pid=S0004-

282X2002000500014\&lng=pt. doi: 10.1590/S0004-282X2002000500014.

ISSN 1695-6141

( ) COPYRIGHT Servicio de Publicaciones - Universidad de Murcia 